\title{
Relationship between Age at Menarche and Metabolic Syndrome in Premenopausal Women: Korea National Health and Nutrition Examination Survey 2013-2014
}

\author{
Yun-Seo Hwang, Eun-Joo Park, Jong-Gi Choi, Hyo-Eun Kim, Seung-Guk Park, Sun-Mi Yoo* \\ Department of Family Medicine, Inje University Haeundae Paik Hospital, Busan, Korea
}

\begin{abstract}
Background: Early menarche may be associated with increased risk of cardiovascular disease. The aim of this study was to evaluate the relationship between age at menarche and metabolic syndrome (MetS) in Korean premenopausal women.

Methods: We used nationally representative data from the Korea National Health and Nutrition Examination Survey from 2013 to 2014, and 3,023 premenopausal women aged 20-55 years were our subjects. We defined early menarche as age at first menstrual period less than 12 years. Multivariable logistic regression analysis was used to evaluate the relationship between age at menarche and MetS after adjusting for current age, and socioeconomic, lifestyle, and reproductive variables.

Results: MetS was much more common in women aged 40-55 years than in women aged $20-39$ years (4.1\% vs. $15.1 \%)$. Compared with women who experienced menarche at age 12-15 years, the risk of MetS in the early menarche group was not higher in either age group, after adjusting for current age, and socioeconomic, lifestyle, and reproductive variables (odds ratio [OR], 1.767; 95\% confidence interval [CI], 0.718-4.351 in those aged 20-39 years; OR, 1.780; 95\% CI, $0.775-4.085$ in those aged $40-55$ years). The risk of MetS in women with menarche at age $\geq 16$ years was not higher than in women with menarche at age $12-15$ years.

Conclusion: Early or late menarche was not associated with an increased risk of MetS in premenopausal Korean women. Even before menopause, current age has a major influence on the development of MetS.
\end{abstract}

Keywords: Menarche; Premenopause; Obesity; Metabolic Syndrome

Received: February 24, 2017, Revised: August 28, 2017, Accepted: October 12, 2017

*Corresponding Author: Sun-Mi Yoo https://orcid.org/0000-0001-7407-8423

Tel: +82-51-797-3220, Fax: +82-51-797-0298, E-mail: syoo@paik.ac.kr 


\section{INTRODUCTION}

Menarche is one of the most important markers of puberty. ${ }^{1)}$ Age at menarche is influenced by several factors, including ethnicity, geography, socioeconomic status, physical activity, and especially, nutrition. ${ }^{1-3)}$ Recently, interest in early menarche is growing due to an increase in precocious puberty. Previous studies suggested that early menarche is associated with an increased risk of breast cancer, cardiovascular disease (CVD), and type 2 diabetes. ${ }^{4-9)}$

Age at menarche in developed countries has decreased from 16.5 years in 1840 to 13.0 years in the 1960s, and has plateaued at approximately 12.5 years. ${ }^{10-13)}$ In Korea, mean age at menarche steadily decreased from 16.59 years for females born in the 1920 s to 12.60 years for those born in the 1990s. Age at menarche significantly decreased in women born after the 1980s. ${ }^{14)}$ Prevalence of childhood obesity also increased during the same period. According to the Korea National Health and Nutrition Examination Survey (KNHANES), childhood obesity has increased from $5.8 \%$ in 1997 to $10.8 \%$ in 2010. Obese children tend to have increased risk for both early menarche and adult obesity, which is strongly associated with an increase in adult CVD. ${ }^{8,15)}$

The metabolic syndrome (MetS) is a group of risk factors for type 2 diabetes and CVD, including hypertension, hyperglycemia, elevated triglycerides, low high-density lipoprotein cholesterol (HDL-C), and central obesity, and is a major predictive variable for CVD. If the age at menarche is one of the factors that can identify the risk of MetS in adulthood, it can be used for early identification of those with risk factors for adult CVD and for prevention of CVD.

However, previous studies in Korean women have shown inconsistent results for the relationship between age at menarche and MetS, and studies mainly focused on postmenopausal women did not reflect recent changes in age at menarche. The aim of this study is to investigate the relationship between age at menarche and MetS in premenopausal women using data from KNHANES 2013-2014 and to determine whether age at menarche is an independent risk factor for MetS and CVD.

\section{METHODS}

\section{Study Population}

This study was conducted using data from KNHANES 2013-2014. The KNHANES is a cross-sectional and nationally representative survey that was designed to evaluate the health and nutritional status in the Korean population of all ages. All participants provided written informed consent, and the institutional review board of the Korea Centers for Disease Control and Prevention approved the study protocol (2013-07CON-03-4C, 2013-12EXP-03-5C). The KNHANES is based on complex, multistage stratified and clustered samples of the Korean population. We selected 3,023 participants among 3,219 premenopausal women aged 20 to 55 years who had complete information on marital status, household income, education level, smoking status, alcohol consumption, physical activity, number of pregnancies, use of oral contraceptives, duration of lactation, and duration of estrogen exposure.

\section{Definitions of Variables}

Participants answered a self-reported questionnaire on their current age, socioeconomic variables (marital status, household income, education level), lifestyle variables (smoking status, alcohol consumption, physical activity), and reproductive variables (number of pregnancies, use of oral contraceptives, duration of lactation). Early menarche was defined as age at the first menstrual period less than 12 years, and late menarche was defined as age at the first menstrual period greater than or equal to 16 years. ${ }^{4,16)}$ Participants were divided into 3 groups based on age at menarche: $<12$ years, $12-15$ years, $\geq 16$ years.

Marital status was divided into two groups. Those who had a spouse or were living with a partner were classified as married, and all others were classified as unmarried. Household income was classified by quartiles based on monthly average family equivalent income: high, higher-middle, lower-middle, and lower. Education level was divided into two groups: high school graduate or less, and college graduate or more.

Smoking status was divided into three groups. (1) Non-smokers were defined as individuals who had never smoked or had smoked less than 100 cigarettes in their lifetime. (2) Past smokers were defined as individuals who had smoked more than 100 cigarettes in the past, but do not currently smoke. (3) Current smokers were defined as individuals who had smoked more than 100 cigarettes and continued to smoke at the time of questionnaire completion. Those who drank more than 5 glasses of alcohol per session and more frequently than twice a week were classified into the high risk drinking group according to the KNHANES recommendations. The physically active group was defined by walking for more than 30 minutes per session and more than five days a week.

The number of pregnancies was divided into two groups, based on pregnancies more than or equal to 3 times, including abortion; use of oral contraceptives was divided into two groups, based on oral contraceptive use for more than 1 month. ${ }^{4)}$ Duration of lactation was divided into 4 groups: $<1$ month, $1-6$ months, $6-12$ months, and $\geq 12$ months. ${ }^{17)}$ Duration of estrogen exposure was calculated from the current age minus the age at menarche.

\section{Definition of Metabolic Syndrome}

Physical examinations and blood tests were performed by the survey team to assess MetS. The body mass index (BMI) was calculated by dividing the measured weight by the square of the height. Plasma glucose, triglycerides, and HDL-C were measured using a Hitachi Automatic Analyzer 7600-210 (Hitachi, Tokyo, Japan) after an 8-12-hour overnight fast. MetS was defined according to International Diabetes Federation and National Heart, Lung, and Blood Institute/American Heart Association guidelines as any three or more of the following: ${ }^{18)}$ (1) waist circumference for Korean women $\geq 85 \mathrm{~cm} ;{ }^{19)}$ (2) fasting triglycerides $\geq 150 \mathrm{mg} / \mathrm{dL}$; (3) HDL-C $<50 \mathrm{mg} / \mathrm{dL}$; (4) systolic blood pres- 
sure $\geq 130 \mathrm{~mm} \mathrm{Hg}$ or diastolic blood pressure $\geq 85 \mathrm{~mm} \mathrm{Hg}$ or taking medicine for high blood pressure; and (5) fasting plasma glucose $\geq 100$ $\mathrm{mg} / \mathrm{dL}$ or taking medicine for high glucose.

\section{Statistical Analysis}

Statistical analysis was performed using sampling weights according to KNHANES guidelines. Because the prevalence of MetS increases as age increases, participants were divided into two groups based on age at the time of the survey: 20-39 years and 40-55 years. In order to compare the characteristics of participants according to age at menarche, continuous variables were reported as mean and standard deviation using analysis of variance, and categorical variables were reported as percentage using the chi-square test. Post-hoc testing using the Bonferroni method was performed to examine differences between the components of MetS in three groups according to the age at menarche. To investigate the independent relationship between age at menarche and MetS, we used multivariable logistic regression analysis. We examined the adjusted odds ratios (ORs) and 95\% confidence intervals (CIs), controlling for current age, socioeconomic status, lifestyle variables, reproductive status, and duration of estrogen exposure. SAS ver. 9.2 (SAS Institute Inc., Cary, NC, USA) was used to perform all statistical analyses. P-values less than 0.05 were considered statistically significant.

\section{RESULTS}

\section{General Characteristics according to the Age at Menarche} Table 1 shows general characteristics according to the age at menarche. The mean current age was 37.29 years, and the mean age at menarche was 13.56 years. Younger women reported earlier menarche (mean current age: 30.98 years with menarche at $<12$ years, 37.09 years with menarche at $12-15$ years, and 43.96 years with menarche at $\geq 16$ years). Women with early menarche were less likely to marry, had more education, and were physically active. They were also less likely to be pregnant 3 times or more and lactate for more than 12 months (Table 1).

\section{Relationship between Age at Menarche and Metabolic Syndrome}

The prevalence of MetS was $8.7 \%$ with menarche at $<12$ years, $8.3 \%$ with menarche at $12-15$ years, and $15.3 \%$ with menarche at $\geq 16$ years.

Table 1 shows that age at the time of survey affected the prevalence and components of MetS. The ages were significantly different among the three groups according to age at menarche. Therefore, we compared the prevalence and components of MetS after dividing the subjects into two groups based on age at the time of survey. Women with early and late menarche were more likely to have MetS after categorization into two groups based on age at the time of survey $(6.0 \%, 3.7 \%$,

Table 1. Characteristics of premenopausal women stratified by age at menarche

\begin{tabular}{|c|c|c|c|c|}
\hline \multirow{2}{*}{ Characteristic } & \multicolumn{3}{|c|}{ Age at menarche (y) } & \multirow{2}{*}{ P-value } \\
\hline & $<12(n=288)$ & $12-15(n=2,321)$ & $\geq 16(n=414)$ & \\
\hline Age at the time of survey (y) & $30.98 \pm 0.60$ & $37.09 \pm 0.23$ & $43.96 \pm 0.60$ & $<0.001$ \\
\hline Age at menarche $(y)$ & $10.74 \pm 0.04$ & $13.44 \pm 0.03$ & $16.76 \pm 0.06$ & $<0.001$ \\
\hline Height (cm) & $160.03 \pm 0.38$ & $160.04 \pm 0.14$ & $158.50 \pm 0.35$ & $<0.001$ \\
\hline Weight (kg) & $59.00 \pm 0.70$ & $57.64 \pm 0.21$ & $57.36 \pm 0.50$ & 0.122 \\
\hline Married & $51.3(3.4)$ & $70.8(1.2)$ & $78.2(2.2)$ & $<0.001$ \\
\hline Household income & & & & 0.683 \\
\hline Low & $8.3(1.8)$ & $7.5(0.7)$ & $9.9(2.1)$ & \\
\hline Mid-low & $25.4(3.0)$ & $25.4(1.2)$ & $25.8(2.6)$ & \\
\hline Mid-high & $30.4(2.8)$ & $32.9(1.2)$ & $34.0(2.6)$ & \\
\hline High & $35.9(3.1)$ & $34.2(1.5)$ & $30.3(2.9)$ & \\
\hline College graduate or more & $61.8(3.3)$ & $48.3(1.3)$ & $29.3(2.3)$ & $<0.001$ \\
\hline Smoking status & & & & 0.737 \\
\hline Never & $86.4(2.3)$ & $88.7(0.8)$ & $87.0(1.7)$ & \\
\hline Past & $6.1(1.5)$ & $4.9(0.5)$ & $5.8(1.3)$ & \\
\hline Current & $7.5(1.7)$ & $6.4(0.6)$ & $7.1(1.3)$ & \\
\hline High-risk drinking & $10.1(1.9)$ & $6.6(0.6)$ & $8.2(1.6)$ & 0.097 \\
\hline Physically active & $43.0(3.2)$ & $37.7(1.1)$ & $31.2(2.4)$ & 0.009 \\
\hline No. of pregnancies $\geq 3$ & $34.9(4.2)$ & $54.1(1.2)$ & $63.4(2.4)$ & $<0.001$ \\
\hline History of oral contraceptive use & $11.8(2.2)$ & $10.3(0.7)$ & $12.6(1.9)$ & 0.437 \\
\hline Duration of lactation & & & & $<0.001$ \\
\hline Never & $57.9(3.2)$ & $43.4(1.2)$ & $34.4(2.8)$ & \\
\hline$<6$ mo & $10.5(1.8)$ & $11.7(0.7)$ & $8.8(1.5)$ & \\
\hline $6-12$ mo & $8.5(1.8)$ & $10.4(0.6)$ & $11.0(1.6)$ & \\
\hline$\geq 12 \mathrm{mo}$ & $23.1(2.9)$ & $34.5(1.1)$ & $45.8(2.8)$ & \\
\hline
\end{tabular}

Values are presented as mean \pm standard error or \% (standard error). Differences between early menarche (or late menarche) and reference group were compared using analysis of variance for continuous variables and the chi-square test for percentage. 
Table 2. Components of metabolic syndrome in premenopausal women by age at menarche and age at the time of survey

\begin{tabular}{|c|c|c|c|c|}
\hline \multirow{2}{*}{ Characteristic } & \multicolumn{3}{|c|}{ Age at menarche (y) } & \multirow{2}{*}{ P-value } \\
\hline & $<12$ & $12-15$ & $\geq 16$ & \\
\hline \multicolumn{5}{|l|}{$20-39 y$} \\
\hline Body mass index $\left(\mathrm{kg} / \mathrm{m}^{2}\right)$ & $23.00 \pm 0.27^{*}$ & $21.86 \pm 0.12^{\dagger}$ & $21.28 \pm 0.36^{\dagger}$ & $<0.001$ \\
\hline Waist circumference $(\mathrm{cm})$ & $75.98 \pm 0.62^{*}$ & $73.08 \pm 0.31^{\dagger}$ & $72.48 \pm 1.01^{\dagger}$ & $<0.001$ \\
\hline HDL cholesterol (mg/dL) & $59.73 \pm 0.95^{\dagger}$ & $58.49 \pm 0.36^{\dagger}$ & $59.40 \pm 1.37^{\dagger}$ & 0.451 \\
\hline Fasting triglycerides (mg/dL) & $90.40 \pm 6.45^{\dagger}$ & $85.69 \pm 1.75^{\dagger}$ & $101.17 \pm 15.07^{\dagger}$ & 0.396 \\
\hline Fasting glucose $(\mathrm{mg} / \mathrm{dL})$ & $90.08 \pm 0.54^{\dagger}$ & $90.63 \pm 0.48^{\dagger}$ & $92.30 \pm 2.63^{\dagger}$ & 0.815 \\
\hline Systolic pressure $(\mathrm{mm} \mathrm{Hg})$ & $104.99 \pm 0.73^{\dagger}$ & $104.47 \pm 0.32^{\dagger}$ & $105.28 \pm 1.02^{\dagger}$ & 0.875 \\
\hline Diastolic pressure $(\mathrm{mm} \mathrm{Hg})$ & $69.67 \pm 0.75^{\dagger}$ & $69.46 \pm 0.29^{\dagger}$ & $68.38 \pm 0.82^{\dagger}$ & 0.401 \\
\hline \multicolumn{5}{|l|}{$40-55$ y } \\
\hline Body mass index $\left(\mathrm{kg} / \mathrm{m}^{2}\right)$ & $24.24 \pm 0.55^{\dagger}$ & $23.42 \pm 0.12^{\dagger}$ & $23.13 \pm 0.18^{\dagger}$ & 0.274 \\
\hline Waist circumference (cm) & $79.43 \pm 1.59^{\dagger}$ & $77.09 \pm 0.33^{\dagger}$ & $76.15 \pm 0.50^{\dagger}$ & 0.204 \\
\hline HDL cholesterol (mg/dL) & $56.81 \pm 2.56^{\dagger}$ & $56.52 \pm 0.40^{\dagger}$ & $56.12 \pm 0.83^{\dagger}$ & 1.000 \\
\hline Fasting triglycerides $(\mathrm{mg} / \mathrm{dL})$ & $101.34 \pm 11.90^{\dagger}$ & $112.25 \pm 2.39^{\dagger}$ & $117.46 \pm 5.71^{\dagger}$ & 0.807 \\
\hline Fasting glucose (mg/dL) & $97.31 \pm 3.65^{\dagger}$ & $96.35 \pm 0.54^{\dagger}$ & $95.74 \pm 1.05^{\dagger}$ & 0.603 \\
\hline Systolic pressure $(\mathrm{mm} \mathrm{Hg})$ & $114.11 \pm 2.30^{\dagger}$ & $111.65 \pm 0.48^{\dagger}$ & $115.45 \pm 1.11^{*}$ & 0.04 \\
\hline Diastolic pressure (mm Hg) & $76.20 \pm 1.81^{\dagger}$ & $73.92 \pm 0.34^{\dagger}$ & $75.78 \pm 0.76^{\dagger}$ & 0.05 \\
\hline
\end{tabular}

Values are presented as mean \pm standard error. Differences between early menarche (or late menarche) and reference group were compared using analysis of variance after adjusting for current age of participants.

HDL, high-density lipoprotein.

*,The same symbols indicate non-significant differences between groups based on the Bonferroni test for analysis of variance.

and $4.2 \%$ in those aged $20-39$ years; $23.4 \%, 14.1 \%$, and $19.2 \%$ in those aged $40-55$ years).

Table 2 shows the distribution of each component of MetS according to the age at menarche after adjusting for current age of participants. In those aged 20-39 years, BMI and waist circumference were significantly higher in women with menarche at $<12$ years of age than in those with menarche at 12-15 years of age or older. HDL-C, fasting triglycerides, fasting plasma glucose, and blood pressures were not significantly different according to age at menarche. In those aged 4055 years, systolic blood pressure was higher in women with menarche at $\geq 16$ years than in those with menarche at $12-15$ years or younger. There were no significant differences in the other components of MetS among the three groups (Table 2).

Table 3 shows the risk of MetS according to the age at menarche after adjusting for confounders. In those aged 20-39 years, compared with women with menarche at 12-15 years, the risk of MetS did not increase in those with early and late menarche after adjusting for current age (OR, 1.922; 95\% CI, 0.951-3.885 with menarche at $<12$ years; OR, 1.053; 95\% CI, $0.317-3.500$ with menarche at $\geq 16$ years). However, in model 2, which was adjusted for marital status, household income, education level, smoking status, high-risk drinking, and physical activity, in addition to current age, the risk of MetS was significantly increased in women with early menarche (OR, 2.035; 95\% CI, 1.0114.094). In model 3 , which was further adjusted for number of pregnancies, history of oral contraceptive use, and duration of lactation, the risk of MetS did not increase in those with early and late menarche (OR, 1.767; 95\% CI, 0.718-4.351 with menarche at < 12 years of age; OR, 0.743 ; $95 \% \mathrm{CI}, 0.171-3.219$ with menarche at $\geq 16$ years of age). In model 4 , which was further adjusted for duration of estrogen exposure, the
Table 3. Risk of metabolic syndrome according to age at menarche and age at the time of survey

\begin{tabular}{|c|c|c|c|}
\hline \multirow{2}{*}{ Characteristic } & \multicolumn{3}{|c|}{ Age at menarche $(y)$} \\
\hline & $<12$ & $12-15$ & $\geq 16$ \\
\hline \multicolumn{4}{|l|}{$20-39$ y } \\
\hline Model 1 & $1.922(0.951-3.885)$ & 1.000 & $1.053(0.317-3.500)$ \\
\hline Model 2 & $2.035(1.011-4.094)$ & 1.000 & $0.931(0.283-3.068)$ \\
\hline Model 3 & $1.767(0.718-4.351)$ & 1.000 & $0.743(0.171-3.219)$ \\
\hline Model 4 & $0.900(0.228-3.560)$ & 1.000 & $1.375(0.320-5.913)$ \\
\hline \multicolumn{4}{|l|}{$40-55$ y } \\
\hline Model 1 & $2.171(0.988-4.772)$ & 1.000 & $1.094(0.773-1.547)$ \\
\hline Model 2 & $2.346(1.094-5.033)$ & 1.000 & $1.005(0.696-1.452)$ \\
\hline Model 3 & $1.780(0.775-4.085)$ & 1.000 & $0.981(0.672-1.434)$ \\
\hline Model 4 & $1.211(0.483-3.035)$ & 1.000 & $1.589(0.682-3.703)$ \\
\hline
\end{tabular}

Values are presented as odds ratio (95\% confidence interval). Odds ratios for metabolic syndrome according to age at menarche; multivariable logistic regression was used. Model 1: adjusted for current age; model 2: adjusted for marital status, household income, education level, smoking status, alcohol consumption, and physical activity, in addition to model 1; model 3: adjusted for number of pregnancies, use of oral contraceptive, and duration of lactation, in addition to model 2; and model 4: adjusted for duration of estrogen exposure, in addition to model 3.

risk of MetS with menarche at $<12$ years of age tended to decrease compared to that in other models, but there was no statistically significant difference. In those aged 40-55 years, the results were the same as in those aged 20-39 years (Table 3 ).

Age at menarche did not show a significant correlation with MetS after analysis of age at menarche as a continuous variable (data not shown). 


\section{DISCUSSION}

We investigated the relationship between age at menarche and MetS in premenopausal Korean women aged 20-55 years using data from KNHANES 2013-2014. Women with early and late menarche did not have increased risk of MetS compared with those who had menarche at 12-15 years of age.

Previous studies have shown inconsistent results for the relationship between age at menarche and MetS. Consistent with our findings, a meta-analysis based on nine articles with prospective studies of both pre- and postmenopausal women showed no association between age at menarche and overall cardiovascular death. ${ }^{16)}$ Cho et al. ${ }^{20)}$ found no association between age at menarche and MetS in postmenopausal Korean women, based on KNHANES 2005. The Japan Collaborative Cohort study also showed no association between age at menarche and mortality from coronary heart disease (CHD) in postmenopausal Japanese women. ${ }^{21)}$

In contrast to our findings, Lim et al. ${ }^{4}$ found that early menarche was associated with an increased risk of MetS in premenopausal Korean women, based on KNHANES 2007-2009. Cho et al. ${ }^{22)}$ also found that early menarche compared with late menarche was associated with increased risk of MetS in premenopausal Korean women, based on KNHANES 2013-2015. A prospective follow-up study of 272 suburban schoolgirls aged between 5-22 and 30-46 years showed that the risk of MetS in adulthood was increased with both early ( $\leq 10$ years) and late ( $\geq 16$ years) menarche. ${ }^{6)}$ A large UK cohort study of women aged 50-64 years showed a U-shape relationship with CHD, and the relative risk of $\mathrm{CHD}$ was lowest for those with menarche at 13 years of age. ${ }^{5)}$

Age, a well-known risk factor for MetS, is also closely related to age at menarche. Mean current age was significantly different by about 6 years according to age at menarche in two previous Korean studies, in contrast to our findings. ${ }^{4,22)}$ Mean current age in our study was also significantly different by 6-7 years according to the age at menarche. In our preliminary analysis, we found that women with menarche before the age of 12 had an increased risk of MetS compared to those who had menarche at 12-15 years of age in all models before classification into two groups based on age at the time of survey (Appendix 1). However, this positive association between age at menarche and MetS disappeared after classification into two groups based on age at the time of survey.

The specific and direct underlying mechanisms involved in the association between age at menarche and MetS remain poorly understood, but it can be inferred that childhood obesity may trigger both early menarche and MetS in later life. Excess adipocytes increase the levels of the adrenal androgen dehydroepiandrosterone and estradiol and reduce the levels of sex hormone-binding globulin (SHBG). ${ }^{23,24)}$ Elevated androgen is highly associated with insulin resistance, and increased plasma insulin levels promote reduction of SHBG levels. ${ }^{25)}$ In other words, higher childhood BMI may contribute to earlier age at menarche and higher adult BMI, which are related to adult MetS and
CVD. ${ }^{8)}$ However, childhood obesity is not the only factor involved in the relationship between early menarche and MetS. In Western countries, the age at menarche has not decreased significantly in the past 20 years, but childhood obesity and adult MetS are steadily increasing. ${ }^{10-13)}$ Further studies are needed to explain the pathophysiological mechanism of the relationship between age at menarche and MetS and to clarify the role of age at menarche on MetS.

This study has several limitations. First, the cross-sectional design of this study prevents determination of the precise causal relationship, although age at menarche precedes MetS. Second, we could not identify a correlation between risk factors at the time of menarche, such as socioeconomic status, BMI, and sex hormone levels, which could contribute to the increase of MetS in adulthood in the early menarche group. Although we controlled a variety of predictable confounding variables, there may still be other confounders that affect MetS, such as genetic background, dietary patterns, and stressful living conditions. Third, there was a limit to measuring the precise duration of estrogen exposure. Therefore, further studies are required to confirm the relationship between duration of estrogen exposure, age at menarche, and MetS.

Despite these potential limitations, this study has several strengths. First, we used the data from KNHANES, a nationally representative survey, to confirm the relationship between age at menarche and MetS. Second, most previous studies on early menarche were conducted in postmenopausal women, but this study reflected the recent changes in age at menarche because our subjects were relatively young women aged 20-55 years before menopause. In particular, we included women born in the late 1980s, when the age at menarche decreased most rapidly in Korea. Finally, in order to reduce age-grouprelated cohort effects on the association between age at menarche and MetS, we performed analyses after dividing subjects into two groups based on age at the time of survey. Our study revealed that the older the women, the more recent the survey, and the younger the birth cohort, the greater the risk of MetS. In other words, individual aging, period influence, and birth cohort turnover may have simultaneously influenced the relationship between the age at menarche and MetS. In order to reflect both aging of individuals and change at a societal level, long-term prospective studies or age-period-cohort analysis of repeated cross-sectional surveys are needed.

In conclusion, early or late menarche was not associated with an increased risk of MetS in premenopausal Korean women. Even before menopause, current age has a major influence on the development of MetS.

\section{CONFLICT OF INTEREST}

No potential conflict of interest relevant to this article was reported.

\section{REFERENCES}

1. Dvornyk V, Waqar-ul-Haq. Genetics of age at menarche: a systematic 
review. Hum Reprod Update 2012;18:198-210.

2. Freedman DS, Khan LK, Serdula MK, Dietz WH, Srinivasan SR, Berenson GS. Relation of age at menarche to race, time period, and anthropometric dimensions: the Bogalusa Heart Study. Pediatrics 2002;110: e43.

3. Wronka I, Pawlinska-Chmara R. Menarcheal age and socio-economic factors in Poland. Ann Hum Biol 2005;32:630-8.

4. Lim SW, Ahn JH, Lee JA, Kim DH, Seo JH, Lim JS. Early menarche is associated with metabolic syndrome and insulin resistance in premenopausal Korean women. Eur J Pediatr 2016;175:97-104.

5. Canoy D, Beral V, Balkwill A, Wright FL, Kroll ME, Reeves GK, et al. Age at menarche and risks of coronary heart and other vascular diseases in a large UK cohort. Circulation 2015;131:237-44.

6. Glueck CJ, Morrison JA, Wang P, Woo JG. Early and late menarche are associated with oligomenorrhea and predict metabolic syndrome 26 years later. Metabolism 2013;62:1597-606.

7. Lakshman R, Forouhi NG, Sharp SJ, Luben R, Bingham SA, Khaw KT, et al. Early age at menarche associated with cardiovascular disease and mortality. J Clin Endocrinol Metab 2009;94:4953-60.

8. Kivimaki M, Lawlor DA, Smith GD, Elovainio M, Jokela M, Keltikangas-Jarvinen L, et al. Association of age at menarche with cardiovascular risk factors, vascular structure, and function in adulthood: the Cardiovascular Risk in Young Finns Study. Am J Clin Nutr 2008;87:187682.

9. Jordan SJ, Webb PM, Green AC. Height, age at menarche, and risk of epithelial ovarian cancer. Cancer Epidemiol Biomarkers Prev 2005;14: 2045-8.

10. Gohlke B, Woelfle J. Growth and puberty in German children: is there still a positive secular trend? Dtsch Arztebl Int 2009;106:377-82.

11. Rigon F, Bianchin L, Bernasconi S, Bona G, Bozzola M, Buzi F, et al. Update on age at menarche in Italy: toward the leveling off of the secular trend. J Adolesc Health 2010;46:238-44.

12. Talma H, Schonbeck Y, van Dommelen P, Bakker B, van Buuren S, Hirasing RA. Trends in menarcheal age between 1955 and 2009 in the Netherlands. PLoS One 2013;8:e60056.

13. Anderson SE, Must A. Interpreting the continued decline in the average age at menarche: results from two nationally representative surveys of U.S. girls studied 10 years apart. J Pediatr 2005;147:753-60.

14. Ahn JH, Lim SW, Song BS, Seo J, Lee JA, Kim DH, et al. Age at menarche in the Korean female: secular trends and relationship to adult- hood body mass index. Ann Pediatr Endocrinol Metab 2013;18:60-4.

15. Freedman DS, Khan LK, Serdula MK, Dietz WH, Srinivasan SR, Berenson GS, et al. The relation of menarcheal age to obesity in childhood and adulthood: the Bogalusa heart study. BMC Pediatr 2003;3:3.

16. Charalampopoulos D, McLoughlin A, Elks CE, Ong KK. Age at menarche and risks of all-cause and cardiovascular death: a systematic review and meta-analysis. Am J Epidemiol 2014;180:29-40.

17. Schwarz EB, Ray RM, Stuebe AM, Allison MA, Ness RB, Freiberg MS, et al. Duration of lactation and risk factors for maternal cardiovascular disease. Obstet Gynecol 2009;113:974-82.

18. Alberti KG, Eckel RH, Grundy SM, Zimmet PZ, Cleeman JI, Donato $\mathrm{KA}$, et al. Harmonizing the metabolic syndrome: a joint interim statement of the International Diabetes Federation Task Force on Epidemiology and Prevention; National Heart, Lung, and Blood Institute; American Heart Association; World Heart Federation; International Atherosclerosis Society; and International Association for the Study of Obesity. Circulation 2009;120:1640-5.

19. Lee SY, Park HS, Kim DJ, Han JH, Kim SM, Cho GJ, et al. Appropriate waist circumference cutoff points for central obesity in Korean adults. Diabetes Res Clin Pract 2007;75:72-80.

20. Cho GJ, Park HT, Shin JH, Kim T, Hur JY, Kim YT, et al. The relationship between reproductive factors and metabolic syndrome in Korean postmenopausal women: Korea National Health and Nutrition Survey 2005. Menopause 2009;16:998-1003.

21. Cui R, Iso H, Toyoshima H, Date C, Yamamoto A, Kikuchi S, et al. Relationships of age at menarche and menopause, and reproductive year with mortality from cardiovascular disease in Japanese postmenopausal women: the JACC study. J Epidemiol 2006;16:177-84.

22. Cho YJ, Lee GH. Association of age at menarche with metabolic syndrome and components of metabolic syndrome in premenopausal women, Korea National Health and nutrition examination survey VI. Int J Diabetes Dev Ctries 2016;36:345-51.

23. Kaplowitz PB. Link between body fat and the timing of puberty. Pediatrics 2008;121 Suppl 3:S208-17.

24. Tchernof A, Despres JP. Sex steroid hormones, sex hormone-binding globulin, and obesity in men and women. Horm Metab Res 2000;32: 526-36.

25. Christakou CD, Diamanti-Kandarakis E. Role of androgen excess on metabolic aberrations and cardiovascular risk in women with polycystic ovary syndrome. Womens Health (Lond) 2008;4:583-94. 
Appendix 1. Risk of metabolic syndrome according to age at menarche

\begin{tabular}{lccc}
\hline & \multicolumn{3}{c}{ Age at menarche (y) } \\
\cline { 2 - 4 } & $<12$ & $12-15$ & $\geq 16$ \\
\hline Model 1 & $2.054(1.204-3.504)$ & 1.000 & $1.109(0.785-1.567)$ \\
Model 2 & $2.194(1.292-3.727)$ & 1.000 & $0.998(0.695-1.432)$ \\
Model 3 & $1.919(1.020-3.608)$ & 1.000 & $0.975(0.673-1.412)$ \\
\hline
\end{tabular}

Values are presented as odds ratio (95\% confidence interval). Odds ratios for metabolic syndrome according to the age at menarche; multivariable logistic regression was used. Model 1: adjusted for current age; model 2: adjusted for marital status, household income, education level, smoking status, alcohol consumption, and physical activity, in addition to model 1; and model 3: adjusted for number of pregnancies, use of oral contraceptive, and duration of lactation, in addition to model 2. 